\title{
Stochastic properties of binocular rivalry alternations
}

\author{
PETER WALKER \\ University College, London, England
}

\begin{abstract}
Previous researches have demonstrated that the successive phase durations in binocular rivalry are independent. These findings are confirmed and extended to chromatic stimuli. The nature of the function that is shown to describe the distribution of the dominance phase durations is consistent with the independence of successive phases and suggests that a parallel may exist between binocular rivalry and the perceptual reversal of ambiguous figures.
\end{abstract}

In three different, though related ways, experiments have indicated that successive rivalry phase durations are independent: (1) Altering the stimulus parameters of just one rivaling field has been demonstrated to have asymmetrical effects on the course of rivalry. Thus, Fox and Rasche (1969) and Levelt (1966) have reported that increasing the stimulus strength (e.g., contrast) of a rivaling image causes a change in the mean duration of dominance of the contralateral image only: the mean duration of dominance of the changed stimulus remains unaffected. (2) Direct statistical tests of sequential dependency have yielded negative results. Blake. Fox, and McIntyre (1971) and Fox and Herrmann (1967) undertook an autocorrelation analysis on the successive phase durations, in addition to determining the average absolute slope of successive durations (a test of first-order dependencies) in a manner outlined by Lathrop (1966). Both procedures failed to provide support for the dependence hypothesis. (3) The nature of the function that has been found to describe the frequency distribution of the rivalry phase durations reasonably well is consistent with the sequential independence of successive phases. Blake et al. (1971), Fox and Herrman (1967), and Levelt (1967) have all demonstrated that a gamma function describes the distribution of dominance phase durations.

The data from two experiments provided an opportunity to test again the notion that the durations of successive rivalry phases are dependent. The first experiment employed rivaling red and green homogeneous fields, and therefore permitted the previous findings (based entirely on rivalry between achromatic stimuli) to be extended to the color domain.

\section{METHODS}

\section{Experiment 1: Chromatic Stimul}

In the first experiment (cf. Walker, 1975, for further details),

This research was undertaken in partial fulfillment of the requirements for the $\mathrm{PhD}$ degree while the author was in receipt of a SRC studentship. The author is indebted to Dr. N. F. Dixon for invaluable supervision, and to $\mathrm{Dr}$. A. R. Jonckheere for help throughout. The author's present address is: Division of Psychology, Preston Polytechnic, Preston PR1 7DP, England. each subject reported the alternations between rivaling red and green homogeneous fields that were presented to his right and left eye, respectively.

Semitransparent tracing-film screens were placed in each field of a modified Unis-France stereoscope. Between these and the right and left eyepieces were placed, respectively, red (Ilford: 205) and green (llford: 625) color filters. While the former transmits freely in the $6.400 \AA$ to infrared range (with heavy absorption at other wavelengths), the latter is restricted to between 5,100 and $5,900 \AA$. Transilluminated by a small fluorescent lamp (opal striplite, $30 \mathrm{~V}$, $30 \mathrm{~W}, 221 \mathrm{~mm}$ ) that was positioned behind and above the stereoscope, the differently colored stereoscope fields served as the rivaling images. The red and green fields were circular in overall shape, and subtended $11 \mathrm{deg} 18 \mathrm{~min}$ and $15 \mathrm{deg} 38 \mathrm{~min}$, respectively. Preliminary experiments demonstrated that providing such a difference in overall size of the two fields served to discourage (a) the two fields from fusing to yield "cortical yellow." and (b) the occurrence of a piecemeal form of rivalry in which different, localized parts of the fields behaved independently. Ignoring the small changes that were made in order to counterbalance eye dominance, the luminance of the two fields measured $1.5 \log \mathrm{fL}$. The fields were positioned so as to appear concentric.

Two microswitches were provided for the subject to report the rivalty alternations. Closure of the switch held in the left hand indicated the dominance of the green field, and vice versa. Each switch was connected to one channel of an Esterline Angus pen recorder.

With regard to the condition relevant to the present analysis, each subject provided data from two separate, 90-sec trials. Although 44 subjects contributed to the total set of data, results from only 33 of these were incorporated in the present analysis, the sole requirement for inclusion being that for each trial the number of alternations was sufficient to permit the type of analysis planned. The 33 subjects were all undergraduate students attending University College London; none were psychologists.

\section{Experimeat 2: Achromatic Stimuli}

In this experiment. subjects reported on the rivalry alternations between competing vertical and horizontally oriented square-wave gratings that were presented to the left and right eyes, respectively (cf. Figure 1).

The two gratings were constructed by applying commercial Letraset to a white, semitransparent surface. Under the conditions of viewing. each "stripe." light and dark alike, subtended $8 \mathrm{~min}$. As in the first experiment, different overall dimensions for the two gratings were employed (cf. Figure 1). Beyond the circular apertures within which the gratings appeared, both visual fields were dark.

Visual stimulation was controlled by a synoptophore that was specially adapted for the experiment. The original light sources were removed from the instrument, the arms extended, and additional lenses employed, appropriate for visual fixation at a distance of $30 \mathrm{~cm}$. As substitutes for the original light sources, two Philips TL $6 \mathrm{~W} / 33$ (cold) lamps were positioned so as to back-illuminate the two gratings. Placed between each light and its 

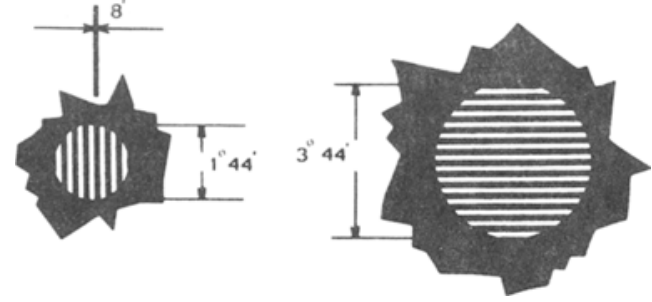

Figure 1. The competing vertical and horizontally orlented square-wave gratings that were presented to the left and right eye, respectively.

grating was an opal perspex diffusing screen. Artificial pupils were added to the synoptophore, and head movements were minimized with chin and forehead rests. The lighter parts of the gratings had a luminance level of approximately $1.5 \log \mathrm{fL}$.

Subjects were provided with a single push button for use with their right hands, and were instructed to press this whenever and for as long as the smaller (vertical) grating was suppressed. On the one 90-sec trial that each subject completed, the responses were recorded in the manner of the first experiment.

Eighteen undergraduate students attending University College served as subjects.

\section{RESULTS AND ANALYSIS}

The results of Experiment 2 (achromatic), although presented with those from the first experiment, receive separate consideration at the end of this section.

With each subject contributing data from two equivalent trials in the first experiment, it was possible to have a replication of each test. As a preliminary to the tests of sequential dependency, the relationship between the standard deviation and mean for the dominance phase durations was expressed as a regression coefficient, and, in accordance with previous findings (Levelt, 1966), a strong positive linear relationship was evident in every case, cf. Table 1. In addition, for each rivaling field, a regression analysis was undertaken to detect temporal linear trends. For this purpose, successive phases of dominance were labeled $1,2,3 \ldots$ and the corresponding durations were regressed on these values. Regression coefficients were determined for each subject together with the associated $F$ values. Presented in Table 2 are the average values obtained for the slopes of the regression lines, and for the associated $F$ values. As can be seen from the table, no significant temporal trends were in evidence.

Several different tests of dependency across successive phases of the rivalry alternations were undertaken.

(1) As a first measure of sequential dependency, the average absolute slope of successive durations (a test of first-order dependencies, cf. Lathrop, 1966) was determined for the red, green, and combined $\mathrm{red} / \mathrm{green}$ sequences. The formula employed in computation was:

$$
L_{j}=\sqrt{\sum_{i=1}^{N-1} \frac{\left|X_{(i+1) j}-X_{i j}\right|}{(N-1) \sigma}} .
$$

where $L_{j}=$ the value of the statistic for the $j$-th sequence, $X_{i j}, X_{(i+1)}=$ successive responses in the $\mathrm{j}$-th sequence, $\sigma=$ the within $\mathrm{js}$ standard deviation.

An analysis combining the red and green phases was made possible with a transformation of the data involving the division of each dominance duration by the mean duration for that field on that trial. This transformation also permitted the data from the different subjects to be later combined.

The expected value for this measure, when successive phase durations are independent, is 1.0 , with an associated standard deviation of $1 / 2 \sqrt{\mathrm{N}}$. A positive relation across successive durations (indicating a stable series) would be reflected in values lower than the expected. Higher values would be indicative of an alternating series.

With the statistic following a normal distribution it was possible to determine a $\mathrm{z}$ score for each of the

Table 1

Mean Values for the Correlation and Regression Coefficients Reflecting the Positive Linear Relation Between the Standard Deviation and Mean of the Dominance Phase Durations

\begin{tabular}{lll}
\hline & & Replicate \\
\hline $\begin{array}{c}\text { Correlation Coefficient } \\
\text { Chromatic Stimuli } \\
\text { Red }\end{array}$ & .802 & \\
$\quad \begin{array}{l}\text { Green } \\
\text { Achromatic Stimuli }\end{array}$ & .925 & .840 \\
$\quad$ Horizontal & .771 & .834 \\
$\quad$ Vertical & .716 & \\
Regression Coefficient & & \\
Chromatic Stimuli & & \\
$\quad$ Red & .629 & .621 \\
$\quad$ Green & .813 & \\
Achromatic Stimuli & & \\
Horizontal & .751 & \\
$\quad$ Vertical & .476 & \\
\hline
\end{tabular}

Table 2

Mean Values for the Regression Coefficients and Associated $F$ Values Reflecting the Absence of Any Temporal Trends in the Sequence of Phase Durations

Replicate

\begin{tabular}{lrl}
\hline $\begin{array}{l}\text { Regression Coefficient } \\
\text { Chromatic Stimuli }\end{array}$ & & \\
$\quad$ Red & .1440 & .0901 \\
$\quad$ Green & .0857 & .0435 \\
$\begin{array}{l}\text { Achromatic Stimuli } \\
\quad \text { Horizontal }\end{array}$ & -.0268 & \\
$\quad \begin{array}{l}\text { Vertical } \\
\text { Associated F Values }\end{array}$ & .0901 & \\
$\begin{array}{l}\text { Chromatic Stimuli } \\
\text { Red }\end{array}$ & & \\
$\quad \begin{array}{l}\text { Green } \\
\text { Achromatic Stimuli } \\
\quad \text { Horizontal }\end{array}$ & .3946 & .2181 \\
$\quad$ Vertical & .1722 & .1812 \\
\hline
\end{tabular}


obtained values. Table 3 gives the average values for the Lathrop statistic and for the corresponding $z$ scores. Combining the two sets of data, the average values obtained for the Lathrop statistic can be seen to be very close to those predicted under the assumption of sequential independence $(0.9993$ for the combined $\mathrm{red} /$ green sequences, 0.9933 for the red, and 0.9919 for the green sequences). Analysis of the chi-squared type on the obtained $z$ values failed to reveal a significant heterogeneity among these values for any set of sequences, and tests involving the conversion of $\Sigma z$ to a unit normal deviate value failed to assign significance to any of the overall deviations from zero. In conclusion, therefore, an analysis based on the Lathrop statistic failed to provide evidence for a sequential dependence within the sequences of rivalry alternations.

(2) Treating the rivalry phase durations as successive values in a time series, an analysis of the type described by Kendall (cf. Kendall, 1973, chap. 2, pp. 21 onwards) was undertaken. One advantage of this analysis over the Lathrop statistic relates to the fact that the expected values have been theoretically, as opposed to empirically determined. Other advantages accrue from its nonparametric nature.

(a) Number of turning points analysis. Defining a turning point as a point in a time series whose associated value has neighboring values less than it (defining a peak) or greater than it (defining a trough), then for a series of $n$ values, the expected number of turning points under the assumption of independence of successive values is $(2 / 3)(n-2)$, and the expected variance of this parameter is then given by $(16 n-29) / 90$. With the number of turning points following a normal distribution, each observed value was converted to a $\mathrm{z}$ score.

An oscillating or rapidly fluctuating series would reflect itself in more turning point than anticipated, while a series tending toward stationarity (successive values being positively correlated) would reflect itself in fewer such points. Thus, the number of turning points was determined for the series from each trial, separately for the exclusively red, the exclusively green, and the combined red/green sequences.

Given in Table 4 are the average values for the $z$ scores associated with the number of turning points observed in each sequence. Analyses of the chi-squared type on the obtained $z$ values failed to reveal a significant heterogeneity among any set of values, and tests involving the conversion of $\sum z$ to a unit normal deviate value failed to assign significance to any of the overall deviations from zero. It may be concluded from these results that the sequential dependence hypothesis obtains no support from the data.

(b) Phase length analysis. Since it is of some interest to consider not just the number of turning points but the distribution of intervals between them, a second related test was performed on the distribution of phase lengths (cf. Wallis \& Moore, 1941). The parts of a time series between successive turning points are referred to as phases (the first and last, incomplete phases are ignored in the analysis), with lengths defined as the number of points spanning a phase, inclusive of the two turning points, less one. Under an assumption of sequential independence, the expected number of phases of length $d$ in a series of total length $n$, is then

$$
\frac{2(\mathrm{n}-\mathrm{d}-2)\left(\mathrm{d}^{2}+3 \mathrm{~d}+1\right)}{(\mathrm{d}+3) !}
$$

A chi-squared test, modified to accommodate the nonindependence of phase lengths from the same series (cf. Wallis \& Moore, 1941) was employed to assess the significance of the observed deviations from the expected frequencies.

Table 5 gives the average observed frequencies for the different lengths of phases (phase lengths of 3 and

Table 3

Mean Values for the Lathrop Statistic and Associated z Scores Reflecting a Sequential Independence Within the Sequences of Dominance Phase Durations

\begin{tabular}{lcc}
\hline & & Replicate \\
\hline $\begin{array}{l}\text { Lathrop Statistic } \\
\text { Chromatic Stimuli } \\
\text { Red }\end{array}$ & & \\
Green & .9745 & 1.012 \\
Red/Green & .9958 & .988 \\
Achromatic Stimuli & .9775 & 1.0212 \\
$\quad$ Horizontal & & \\
Vertical & 1.039 & \\
Horizontal/Vertical & 1.059 & \\
Associated z Values & 1.002 & .080 \\
Chromatic Stimuli & & -120 \\
Red & & .169 \\
Green & -.200 & \\
Red/Green & -.030 & \\
Achromatic Stimuli & -.1615 & \\
Horizontal & .280 & \\
Vertical & .420 & \\
Horizontal/Vertical & .244 & \\
\hline
\end{tabular}

Table 4

Mean Values for the $z$ Scores Associated With the Number of Turning Points Analysis

\begin{tabular}{lcc}
\hline & Replicate \\
\hline z Values \\
$\begin{array}{l}\text { Chromatic Stimuli } \\
\text { Red }\end{array}$ & .00 & .02 \\
Green & .12 & -.04 \\
Red/Green & .20 & .25 \\
Achromatic Stimuli & & \\
$\quad$ Horizontal & .13 & \\
Vertical \\
Horizontal/Vertical & .48 & \\
\hline
\end{tabular}


Table 5

The Mean Observed Frequencies for the Different Phase Lengths Together With the Mean Expected Frequencies

\begin{tabular}{|c|c|c|c|c|}
\hline & \multirow{2}{*}{$\begin{array}{l}\text { Phase } \\
\text { Length }\end{array}$} & \multicolumn{2}{|c|}{ Frequency } & \multirow{2}{*}{$\begin{array}{l}\text { Chi- } \\
\text { Squared }\end{array}$} \\
\hline & & Obs'd & Exp'd & \\
\hline \multicolumn{5}{|c|}{ Chromatic Stimuli } \\
\hline & 1 & 5.15 & 4.89 & \\
\hline Red & 2 & 1.87 & 1.97 & 1.08 \\
\hline & 3 & .51 & .63 & \\
\hline & 1 & 4.66 & 4.78 & \\
\hline Green & 2 & 2.33 & 1.92 & 4.79 \\
\hline & 3 & .39 & .61 & \\
\hline & 1 & 12.03 & 11.45 & \\
\hline Red/Green & 2 & $\begin{array}{l}4.63 \\
170\end{array}$ & 4.85 & 1.26 \\
\hline \multirow{2}{*}{\multicolumn{5}{|c|}{ Replicate }} \\
\hline & 1 & & & \\
\hline Red & 2 & 2.00 & 2.03 & .07 \\
\hline & 3 & .63 & .65 & \\
\hline & 1 & 4.96 & 4.95 & \\
\hline Green & 2 & 1.96 & 1.99 & .19 \\
\hline & 3 & .57 & .63 & \\
\hline & 1 & 13.00 & 11.83 & \\
\hline Red/Green & 2 & 4.57 & 5.02 & 4.69 \\
\hline & 3 & 1.66 & 1.80 & \\
\hline \multicolumn{5}{|c|}{ Achromatic Stimuli } \\
\hline \multirow{4}{*}{ Horizontal } & 1 & 4.38 & 4.07 & \multirow{4}{*}{.48} \\
\hline & 2 & 1.50 & 1.60 & \\
\hline & 3 & .50 & .50 & \\
\hline & 1 & 5.11 & 4.14 & \\
\hline \multirow[t]{3}{*}{ Vertical } & 2 & 1.61 & 1.63 & \multirow[t]{3}{*}{5.12} \\
\hline & 3 & .27 & .51 & \\
\hline & 1 & 8.38 & 9.76 & \\
\hline \multirow[t]{2}{*}{ Horizontal/Vertical } & 2 & 4.33 & 4.11 & 4.22 \\
\hline & 3 & 1.72 & 1.41 & \\
\hline
\end{tabular}

Note. Also tabled are the corresponding chi-squared values, the value required for significance being 5.99.

over being pooled) together with the average expected values. The associated values for chi squared are also tabled, these values being derived from the "total" frequencies, obtained by adding the results across subjects. As can be seen from the table, the observed frequencies failed to deviate significantly from those expected on the assumption of independence.

(3) In order to assess the effects of increasing the separation between "successive" phase durations, an autocorrelation analysis was performed, through lag 5. Apart from revealing the effects of temporal separation, such an analysis, unlike the preceding ones, allows the correlation between successive red and preceding green phases to be separately considered from that between successive green and preceding red. The following four phase relations could therefore be considered : (a) red with preceding green, (b) green with preceding red, (c) red with preceding red, and (d) green with preceding green.

Given in Table 6 are the average values obtained for the autocorrelation coefficients for each of the four possibilities. Figure 2 illustrates the same data in the form of correlograms, with the inclusion of the mean standard deviation associated with these average values (the vertical bars delineate the range of values spanned by two standard deviations, symmetrically placed about the zero level).

After what would be an initial coefficient of 1.0 for lag zero, the coefficients can be seen to be of small magnitude, reflecting the absence of any sequential dependence.

Inspection of the correlograms suggests, however (though for the "original" data only), the existence of a downward trend across successive lags. Formal tests (cf. Jonckheere \& Bower, 1967) assigned significance to these trends for all four phase relations in the original data (the associated $\mathrm{z}$ scores being 2.259 , $3.28,1.66$, and 1.66 for the correlations $a$ to $b$, respectively), though for none in the replicate data (the $\mathrm{z}$ scores in this case being $-0.98,0.98,1.57$, and 0.725). However, in view of the considerable individual differences in trend that were present, it is unfortunate that formal tests for heterogeneity were precluded by the rather short nature (only five values) of the protocols. As Jonckheere and Bower point out, the chi-squared test suggested for this purpose is really only applicable where the individual protocols involve at least 10 values. Moreover, although the trends in the one set " of data are statistically significant, it must be remembered that correlation coefficients of this order attribute only a very small portion of the total variance to the correlation (cf. Hays, 1963 , p. 502 , who points out that a correlation coefficient of 0.15 , say, could be interpreted as attributing only $2.25 \%$ of the total variance to a linear correlation). Together with the complete absence of such trends in the replicate data, it would seem that

Table 6

Mean Values for the Autocorrelation Coefficients, Through Lags 1-5, Between RG-Successive Red and Preceding Green Phases, GR-Successive Green and Preceding Red Phases, Etc.

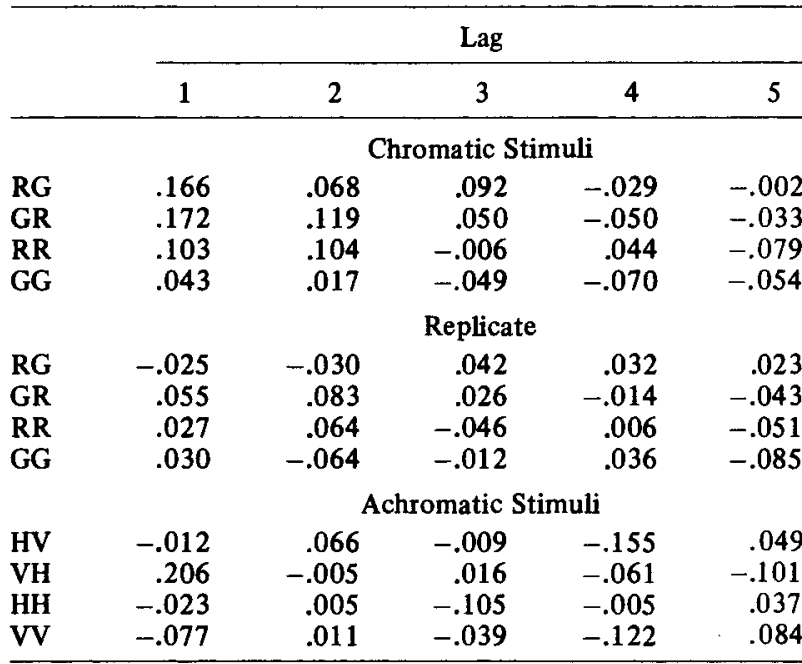

Note. $H=$ horizontal grating and $V=$ vertical grating. 

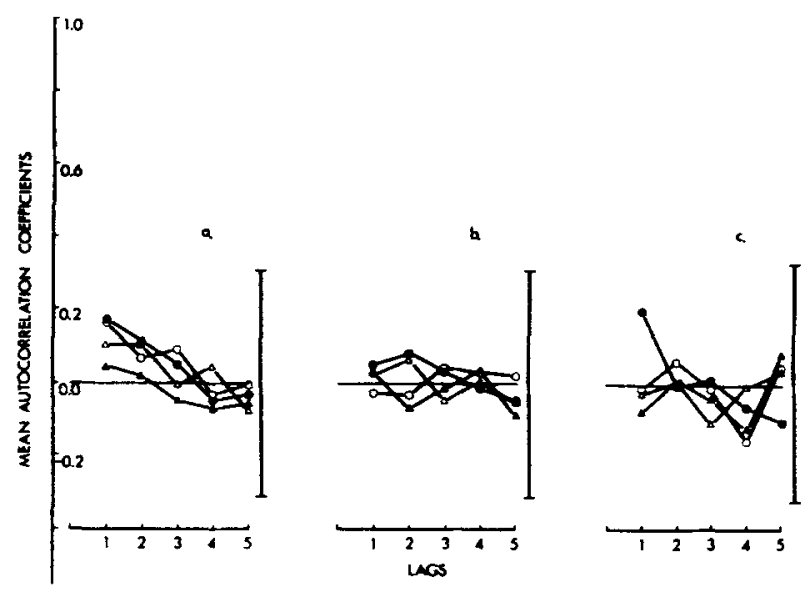

Figure 2. The mean autocorrelation coefficients that are presented in Table 6 are here illustrated in the form of correlograms. The vertical bars delimit the range of values spanned, for both positive and negative coefficients, by the mean of the standard deviations associated with each of the obtained values. a and $b=$ chromatic stimuli, $b=$ replicate data, $c=$ achromatic stimuli. $O-=R G$ for a and $b$, and $=$ HV for c; $-=$ GR for a and $b$, and $=V H$ for $c ; \Delta=R R$ for $a$ and $b$, and $=$ HH for $c$; $\Delta-=G G$ for $a$ and $b$, and $=V V$ for c. cf. Table 6 for symbol code.

whatever processes underlie the consistent behavior, across successive lags, of the autocorrelation coefficients, they do not contribute "significantly" to the occurrence or temporal course of binocular rivalry.

(4) Finally, the frequency distributions of the rivalry phase durations were compared with a theoretical frequency distribution. For this purpose, the values for the phase durations were converted to histogram form, being assigned to intervals of $0.2 \mathrm{sec}$ width.

In Figure 3. the frequency values for the phase durations, combined over subjects, are compared with the frequencies generated by the two-parameter gamma distribution:

$$
f(x)=\frac{\lambda^{r}}{(r-1) !} x^{r-1} e^{-\lambda x}
$$

As mentioned above, to establish a common baseline across subjects, the individual dominance phase durations of a field were divided by the mean duration for that field on that trial. Such a procedure obtains a mean equal to 1.0 . The mean and variance of the pooled frequency distributions were used to estimate the parameters $r$ and $\lambda$. on the basis of the relationship:

$$
\lambda=\mathrm{x} / \mathrm{o}^{2} \text { and } \mathrm{r}=\mathrm{x}^{2} / \mathrm{o}^{2} .
$$

The computed value for $r$ was rounded off to the nearest integer before being used in the gamma function.
The graphic fit of the frequency distributions can be seen to be close, indicating again that successive rivalry phase durations are independent. Moreover, the actual values obtained for $r$ and $\lambda$ are directly comparable to those obtained in previous studies (Blake et al., 1971; Fox \& Herrmann, 1967; Levelt, 1966). The significance of this striking correspondence is difficult to interpret.

\section{Achromatic Stimuli}

As can be seen from Tables 1-6, similar conclusions may be drawn concerning the achromatic stimuli, that is, regarding the alternation process between the differently oriented square-wave gratings. There was again the strong positive linear relationship between the standard deviation and mean value for the dominance phase durations, together with an absence of temporal trends across successive phases.

The Lathrop statistic failed to attach significance to the results, and the same story held for the number of turning points analysis. From Table 5 , it can be seen that the analysis based on the phase lengths similarly failed to yield significant results. The autocorrelation analysis, cf. Table 6 , yielded small insignificant average values, with perhaps the one exception of the autocorrelation of vertical with preceding horizontal phases, for lag 1. Finally, inspection of Figure 3 indicates that the two-parameter gamma function again described reasonably well the frequency distribution of the rivalry phase durations, for both stimulus fields.

In summary, therefore, it would seem justifiable to conclude, as with the chromatic stimuli, that whatever processes might give rise to a statistical dependence across successive phase durations in rivalry, they do not contribute significantly to the occurrence or temporal course of rivalry.

\section{DISCUSSION}

For none of the several tests employed was there evidence to suggest that successive binocular rivalry phases are dependent, for either achromatic or chromatic stimuli.

On this basis, it is tempting to reject explanations of the rivalry process that hold that the alternations arise from the adaptation toward the currently dominant stimulus, and the recovery from adaptation toward the nondominant stimulus (the traditional explanation of the reversal of ambiguous figures, cf. Orbach. Erlich, \& Heath, 1963), since, for example, longer periods of red dominance may then be expected to be succeeded by longer periods of green dominance. However, a number of additional postulates need to be incorporated before such a model can begin to describe the rivalry process. and indeed. there are a variety of facts that speak against involving adaptation in this way. Adaptation may, 

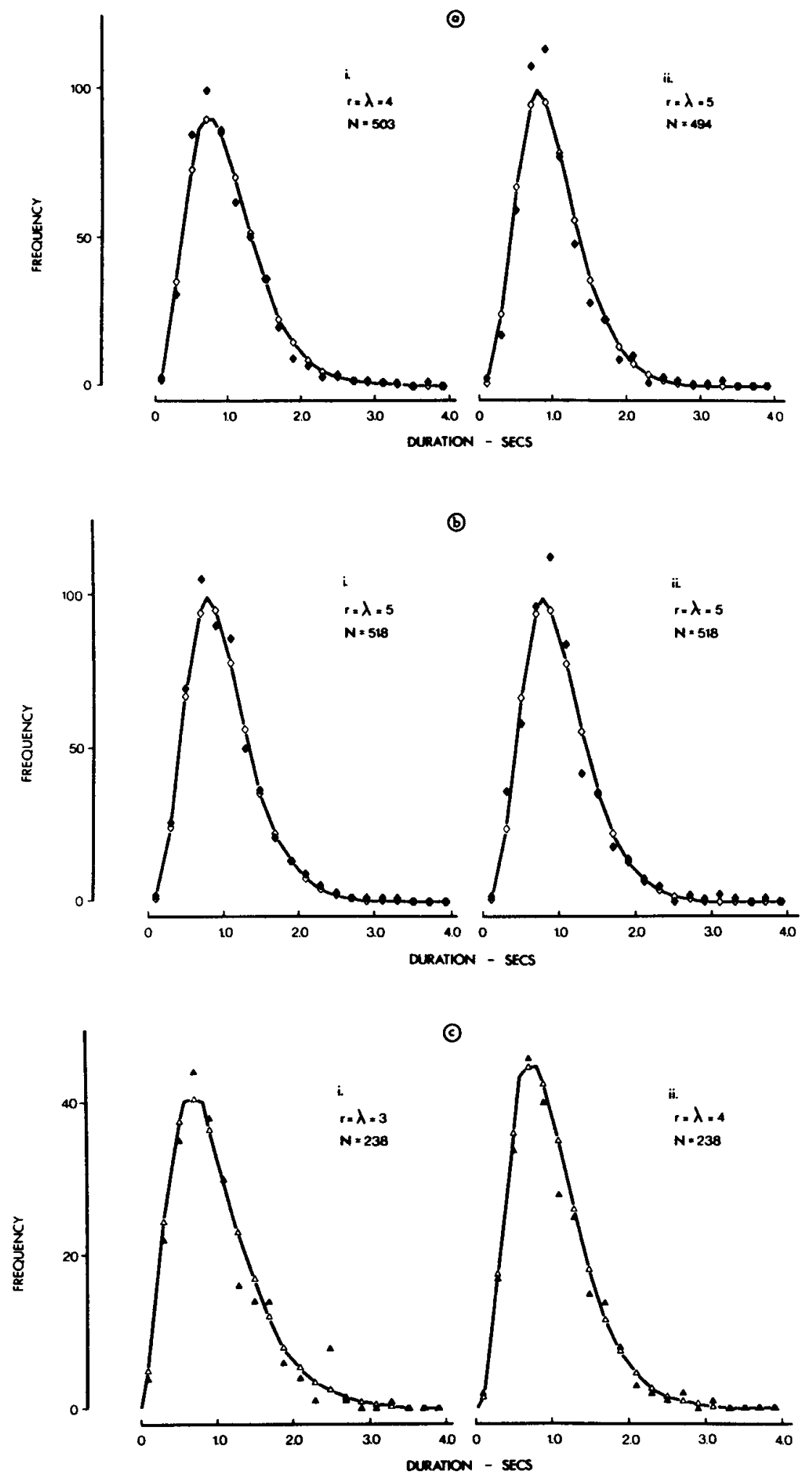

Figure 3. $1-\Delta-$, the observed frequencies, combined over subjects, associated with the different phase durations, are compared with the frequencies generated by the gamma distribution, $\Delta-\Delta-$, having the values indicated for $r$ and $\lambda$. a and $b$, chromatic stimuli, with $i$ and if corresponding to the dominance phases of the red and green fields, respectively. b, replicate data. c, achromatic stimuli, with i and if correaponding to the dominance phases of the vertical and horizontal gratings. 
however, be assigned a more indirect role in a model that accommodates the property of sequential independence, as Taylor and Aldridge (1974) have outlined with reference to the alternation of ambiguous figures.

Finally, it is interesting that the perceptual alternations of a variety of ambiguous figures display the same stochastic properties as rivaling images. Borsellino, De Marco, Allazetta, Rinesi, and Brartolini (1972) have shown that the same two-parameter gamma distribution describes the reversals of the Necker cube and Schroeder staircase. Moreover, employing an autocorrelation analysis, these authors demonstrated more directly that the successive reversals are independent. Although not proof that the same mechanisms underlie the perceptual alternations in the two contexts, this similarity does suggest that the processes underlying rivalry may be more general than is traditionally thought, and not at all restricted to conditions that involve the separate presentation of different stimuli to the two eyes.

\section{REFERENCES}

Blake, R. R., Fox, R., \& MclNTyRe, C. Stochastic properties of stabilised-image binocular rivalry alternations. Journal of Experimental Psychology, 1971, 88, 327-332.

Borsellino, A., De Marco, A., Allazetta, A., Rinesi, S., \& Bartolini, B. Reversal time distribution in the perception of visual ambiguous stimuli. Kybernetic, 1972, 10, 139-144.
Fox, R., \& Herrmann, J. Stochastic properties of binocular rivalry alternations. Perception \& Psychophysics, 1967, 2, 432.436.

Fox, R., \& RASChE, F. Binocular rivalry and reciprocal inhibition. Perception \& Psychophysics, 1969, 5, 215-217.

HAYs, W. L. Statistics. London: Holt, Rinehart \& Winston, 1963.

JonCKHEERE, A. R., \& Bower, G. H. Non-parametric trend tests for learning data. British Journal of Mathematical and Statistical Psychology, 1967, 20, 163.

Kendall, M. G. Time series. London: Charles Griffin, 1973.

LA THR OP, R. G. First-order response dependencies at a differential brightness threshold. Joumal of Experimental Psychology, 1966, 72, 120-124,

LEVELT, W. J. M. The alternation process in binocular rivalry. British Journal of Psychology, 1966, 57, 225-238.

Levelt, W. J. M. Note on the distribution of dominance times in binocular rivalry. British Joumal of Psychology, 1967, 58, 143-145.

Orbach, J., ERLICh, D., \& Heath, H. A. Reversibility of the Necker cube: I. An examination of the concept of "satiation of orientation." Perceptual and Motor Skills, $1963,17,439-458$.

TAYlor, M. M., \& Aldridge, K. D. Stochastic processes in reversing figure perception. Perception \& Psychophysics, $1974,16,9-27$.

WALKER, P. S. The subliminal perception of movement and the "suppression" in binocular rivalry. British Journal of Psychology, $1975,66,347-356$.

W Allis, W. A., \& Moore, G. H. A significance test for timeseries analysis. Journal of the American Statistical Association, $1941,36,401$.

(Received for publication April 1, 1975; revision received August 21, 1975.) 